\title{
On the Consistency of Highly Accurate QCBED Data Refined from Different Zone Axes for Ferromagnetic Tetragonal $\mathrm{L1}_{0}$ FePd
}

\author{
X. H. Sang, A. Kulovits, J. M. K. Wiezorek \\ Department of Mechanical Engineering and Materials Science, Swanson School of Engineering, \\ University of Pittsburgh, 848 Benedum Hall, 3700 O Hara Street, Pittsburgh, PA 15261
}

For the investigation of intrinsic properties of the ferromagnetic tetragonal $\mathrm{L}_{0}$ ordered phases $\gamma_{1^{-}}$ FePd and $\gamma_{2}$-FePt (Fig. 1) the electronic structures of both phases should be determined by experiment and/or theory. Despite the fact, that Pd and Pt belong to the same column of the periodic table of elements and $\gamma_{1}$-FePd and $\gamma_{2}$-FePt are structurally very similar, these phases exhibit very different solid solution behavior. This renders them useful as model systems for the study of $3 d-4 d$ and $3 d-5 d$ electron interactions in transition metal compound systems, which currently are not robustly and accurately described using ab initio calculations. Quantitative convergent beam electron diffraction (QCBED) in combination with energy filtered transmission electron microscopes (EFTEM) provides methods to obtain experimental data on the electronic structure of crystals suitable for validation of electronic structure based calculations [1]. Experimental determination of the electron density distribution by CBED requires very precise and accurate structure factor measurements [1]. This includes CBED pattern acquisition and computational refinement. Here, we used a JEOL JEM2100F with a Tridiem GIF for QCBED experiments, using a new off-zone axis multi-beam QCBED method, developed and recently applied successfully to $\mathrm{Si}$ and $\mathrm{NiAl}$ [2], to obtain highly accurate DW factors and structure factors simultaneously and to investigate $\gamma_{1}$-FePd and $\gamma_{2}$-FePt. In this method, the sample is tilted off zone axis such that three or five reflections intersect the Ewald sphere exactly. This ensures that those three or five beams are strongly excited and renders the CBED pattern very sensitive to change in structure factors. Unlike the systematic row and the zone axis method for QCBED [2], the current method provides sufficient sensitivity to changes of Debye Waller (DW) factors and structure factors to facilitate studies on FePd and FePt.

Low order structure factors up to 222 for FePd have been accurately measured here by acquisition and refinement of CBED patterns near different zone axes orientations at $-153^{\circ} \mathrm{C}$. The difference (Fig. 2a) between the measured X-ray structure factors and independent atom model (IAM) structure factors calculated from Doyle and Turner [3] shows the influence of bonding between Fe atom and Pd atom on low order structure factors. Theoretically, structure factors measured from different zone axes should be the same. Our data (Fig. 2a) shows that some low order structure factors from different zone axes are consistently significantly different, based on the statistical error of our measurements. Low order structure factors for $\mathrm{L} 1_{0}$ ordered $\gamma$ - TiAl, which is non-ferromagnetic and isostructural to $\gamma_{1}-\mathrm{FePd}$ and $\gamma_{2}-\mathrm{FePt}$, were obtained from different zone axes using the same off-zone axis multi-beam QCBED method. The data summarized in Fig. $2 b$ shows that the structure factors of $\mathrm{L} 1_{0}$ ordered $\gamma-\mathrm{TiAl}$ from different zone axes are consistent within the statistical error of the measurement. We attribute the enhanced scatter of the $\mathrm{L} 1_{0}$ ordered $\gamma_{1}-\mathrm{FePd}$ to its ferromagnetic nature, since the experimental conditions were the same as used for the $\gamma-$ TiAl measurements. We plotted the relationship between the refined structure factor and the angle, $\theta$, between the incident beam and the 'easy' magnetization axis [001] for $\mathrm{L} 1_{0}$ ordered $\gamma_{1}$-FePd (Fig. 3). The refined structure factor changes linearly with the sine or cosine of the angle $\theta$. It appears that the linear relationships observed here could be related to the interaction of the electron beam with the magnetic fields of the investigated crystal volumes. Such possible effects from crystal magnetization in structure factor 
measurments have not been observed before since hard ferromagnetic materials have rarely been investigated by QCBED. Conventionally the interaction between fast electrons and a periodic potential is described by the Bloch wave formalism, which does not include a magnetic term. Here, inspired by the accurate results from our QCBED experiments, we speculate that the current FePd structure factor data can provide us with experimental data to establish the Bloch wave formalism for magnetic materials. Correction of QCBED data for magnetic materials is essential for understanding bonding behaviors of magnetic intermetallics and the associated d-electron interactions.

We acknowledge support from the Office of Science, Office of Basic Energy Sciences, Division of Materials Sciences and Engineering, U.S. Department of Energy under contract DE-FG0208ER46545, and access to facilities of the Materials Micro-Characterization Laboratory of the Department of Mechanical Engineering and Materials Science, University of Pittsburgh.

\section{References}

[1] J.M. Zuo et al., Nature 401 (1999) 49.

[2] X.H. Sang et al., Acta Cryst. A66 (2010) 694.

[3] P.A. Doyle et al., Acta Cryst. A24 (1968) 390.
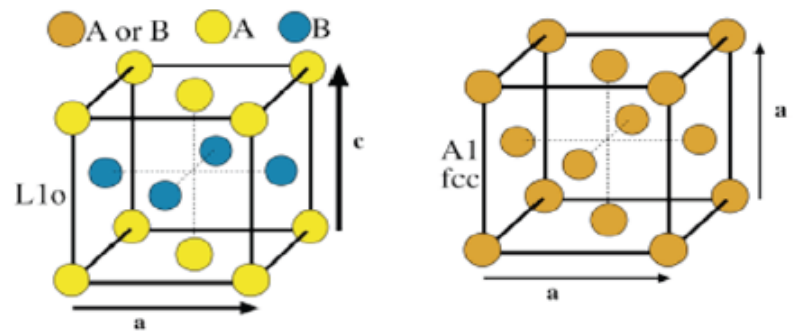

FIG. 1. Conventional unit cell representations of the chemically ordered, $\mathrm{L}_{0}$, and chemically disordered, A1, crystal structures of the A-B compounds FePd, FePt and TiAl.
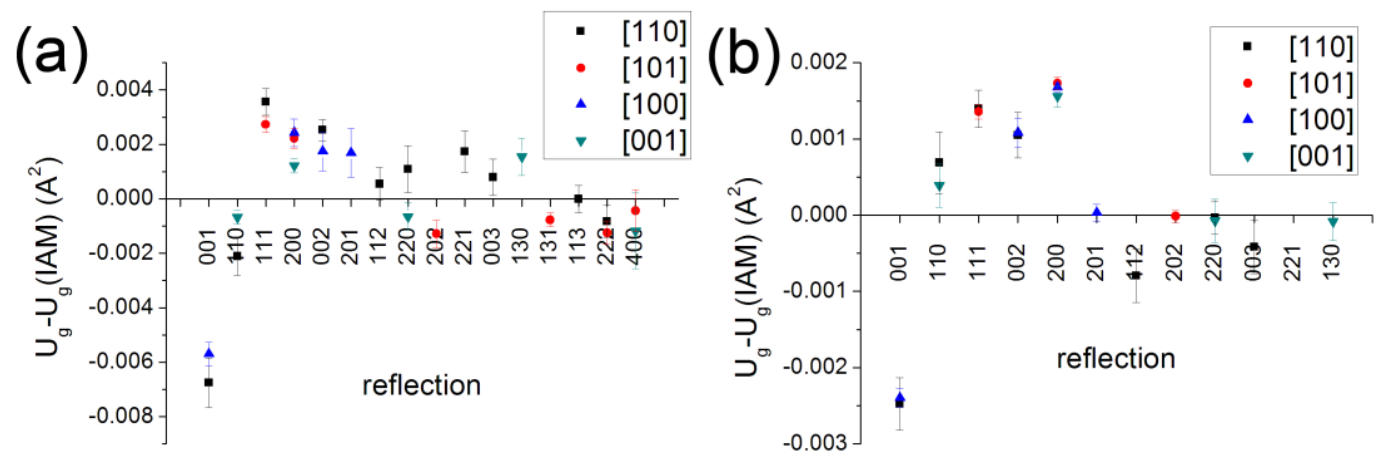

FIG. 2. Difference between CBED measured and IAM structure factors. (a) FePd, (b)

TiAl.

FIG. 3. Refined structure factors ((a) 110, (b) 200) versus angle between the incident beam and the easy magnetization axis [001].
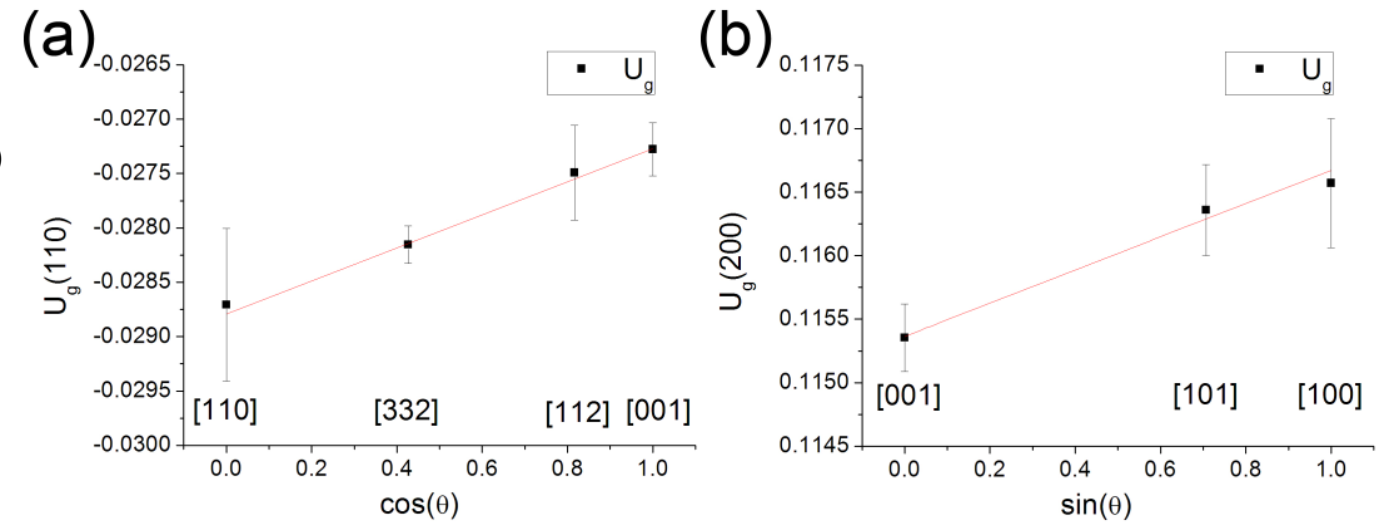